\title{
Una propuesta de complementariedad entre los estudios de migración y psicoanalíticos en salud mental
}

\section{A proposal for complementarity between migration and psychoanalytical studies in mental health}

Recibido el 20 de marzo de 2019. Aceptado el 25 de octubre de 2019. Publicado el 6 de noviembre de 2019.

*Autor para correspondencia: Ángel Luna, correo electrónico, angellunadem2016@colef.mx

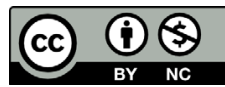

Esta obra está protegida bajo una Licencia Creative Commons Atribución-NoComercial 4.0 Internacional.
Ángel Luna ${ }^{a}$ iD https://orcid.org/0000-0003-3734-7000 Silvia Mejia-Arango ${ }^{b}$ (D) https://orcid.org/0000-0002-6416-1159

\footnotetext{
${ }^{a}$ El Colegio de la Frontera Norte, Doctorado en Estudios de Migración, Tijuana, Baja California, México, correo electrónico: angellunadem2016@colef.mx

${ }^{\text {b }}$ El Colegio de la Frontera Norte, Departamento de Estudios de Población, Tijuana, Baja California, México, correo electrónico: smejia@colef.mx
}

\section{Resumen:}

El objetivo de este artículo es discutir los fundamentos epistemológicos de los estudios psicoanalíticos y su posibilidad de complementariedad con los estudios de migración basados en la sociología. La metodología utilizada fue la revisión de producción teórica sobre el tema de epistemología en psicoanálisis y la tradición de investigación en sociología, así como sobre estudios de migración enfocados en salud mental. Entre los resultados, se encontraron importantes coincidencias epistemológicas y metodológicas entre ambas disciplinas. A partir de eso, se presenta una breve propuesta que pone en evidencia la viabilidad de generar estudios complementarios sobre migración, específicamente en contextos fronterizos. Se sostiene que el valor de este artículo radica en la exploración de una posibilidad metodológica poco desarrollada en los estudios de migración, la cual profundiza en aspectos subjetivos e intersubjetivos en la experiencia de migrar. Se considera que dicha propuesta puede fungir como guía para estudios posteriores.

Palabras clave: Epistemología, migración, salud mental, psicoanálisis, subjetividad.

\begin{abstract}
:
The objective of this article is to discuss the epistemological foundations of psychoanalytic studies and their possibility of complementarity with migration studies based on sociology. The methodology used was the review of theoretical production about epistemology in psychoanalysis, the tradition of research in sociology, as well the migration studies focused on mental health. Among the results, important epistemological and methodological coincidences between both disciplines were found. From this, a brief proposal is presented
\end{abstract}

CÓMO CITAR: Luna, Á. y Mejia-Arango, S. (2019). Una propuesta de complementariedad entre los estudios de migración y psicoanalíticos en salud mental. [A proposal for complementarity between migration and psychoanalytical studies in mental health]. Estudios Fronterizos, 20, e034. doi:https://doi.org/10.21670/ref.1913034 
that highlights the feasibility of generating complementary studies on migration, specifically in border contexts. It is argued that the value of this article lies in the exploration of a methodological possibility little developed in migration studies, which delves into subjective and intersubjective aspects in the experience of migration. It is considered that this proposal can serve as a guide for further studies.

Keywords: Epistemology, migration, mental health, psychoanalysis, subjectivity.

\section{Introducción}

El mundo objetivo ha sido el más investigado en los estudios de migración. Esto es evidente en abordajes que van desde las teorías sobre las causas de la migración, hasta aquellas que se concentran en su continuidad, integración social y económica de los sujetos migrantes, así como también, las teorías sobre su incorporación laboral. Sin embargo, y a pesar de que estas investigaciones explican ampliamente el fenómeno migratorio, se considera relevante identificar la medida en que son integrados los aspectos subjetivos inherentes en la experiencia de migrar. Por ejemplo, se ha identificado que, en algunos casos, los sujetos migrantes ocupan puestos de trabajo por debajo de sus expectativas, así como también, llegan a establecerse en condiciones precarias, viviendo bajo persecución, de manera ilegal o permaneciendo con malestares emocionales (Sarzuri-Lima, 2013).

Los contextos fronterizos en términos geopolíticos, son regiones donde se vuelven palpables las secuelas de los procesos migratorios. Son espacios donde se vive la experiencia de la adaptación, la del ajustarse con valijas que no solo contienen pertenencias, sino una serie de experiencias de vida. Cuando un sujeto emigra, se enfrenta a trabajos de elaboración emocional que no siempre son exitosos y que, en muchas ocasiones, derivan en una nostalgia que impide la integración al lugar de destino (Piastro, 2015). Por lo tanto, es un hecho que en los escenarios migratorios no solamente nos encontramos con situaciones o hechos objetivables, sino también, a sujetos con experiencias que involucran conflictos internos o mentales, mismos que se vuelven un reto y que exigen metodologías adecuadas para su comprensión.

En ese sentido, se ha planteado que el proceso de migración en sí mismo puede estar vinculado con el origen de trastornos mentales. Se ha distinguido que el sujeto migrante atraviesa por un estado de vulnerabilidad psicosocial que se vuelve comprensible al plantear el acto migratorio como un proceso, esto es, a partir de tres etapas: a) pre-migración, b) migración y c) post-migración (Bhugra, 2004).

Es importante destacar la existencia de investigaciones que han identificado una vulnerabilidad psicosocial experimentada por la población migrante, en ellos se han integrado aspectos de la experiencia subjetiva de quien migra, destacándose que, si bien no todos sufren de desajustes importantes en términos de salud mental, sí lo experimentan un porcentaje importante de ellos (Achotegui, 2004; Ingleby, 2008). Sin embargo, es claro que esos estudios se concentran tanto en la explicación de reacciones, así como, en algunos casos, la posible aminoración de malestares emocionales acaecidos en el lugar de destino, o esquemáticamente hablando, en la tercera etapa del proceso migratorio. Esto no se considera inadecuado, pero sí limitado pues, aunque se aborden las experiencias previas a la migración de tipo traumáticas, la comprensión está dirigida a las explicaciones y ajustes en el lugar de destino, sin 
averiguar a fondo sobre el desajuste interno, el cual puede ser de mayor impacto, así como de origen muy anterior y permanente, requiriéndose una comprensión que integre elementos psicosociales de experiencias originarias para su análisis. Con esto se hace referencia a que cuando en las investigaciones se ha buscado comprender el sufrimiento o experiencia de padecimientos en sujetos migrantes, se ha puesto poca o nula atención a la comprensión de experiencias psicosociales y constructoras de la personalidad previas a la migración.

Pareciera fuera de lugar y sin relevancia el considerar que los aspectos que dieron origen y conformaron el funcionamiento mental de los sujetos migrantes, siguen impactando en el presente, aun cuando fueron generados en un pasado remoto. Pareciera también, que se comprende muy poco sobre el funcionamiento inconsciente en el que, paradójicamente, el propio sujeto migrante pudiera no tener conocimiento total, ni control completo. Con esto se hace referencia al estudio de elementos ocultos pero descifrables a través de metodologías específicas como la planteada por los estudios psicoanalíticos, a través de la cual, se explora la historia de los sujetos desde su propia subjetividad. Esto es, a través de la escucha, desde sus propias palabras, desde sus experiencias, sus significados, sus fantasías y sus pensamientos dinamizados a nivel inconsciente, pero a la vez, ligados a elementos socioculturales de un ambiente pasado en el que los sujetos se han desarrollado.

Ahora bien, y considerando a lo fronterizo desde una perspectiva metafórica, es posible considerar que también existen fronteras en los ámbitos académicos, las cuales, llevan a concentraciones exclusivas y separaciones entre disciplinas. Estas fronteras son claras en los estudios de migración y en los estudios psicoanalíticos. Sin embargo, al estudiar toda manifestación psíquica humana, los estudios psicoanalíticos están llamados a cruzar fronteras teóricas y vincularse en un espacio donde confluyen objetos de estudio comunes, por ejemplo, las personas migrantes. Esta situación les lleva al límite con los estudios de migración. Si se busca una comprensión del fenómeno migratorio de manera amplia y en integración con elementos subjetivos e intersubjetivos de quienes migran, ambas disciplinas están llamadas a buscar el intercambio en complementación.

Histórica y epistemológicamente hablando, la investigación psicoanalítica se ha dirigido desde una perspectiva más psicopatológica hacía una exploración de fenómenos humanos que involucran a otras disciplinas, tales como la sociología y la antropología. Para Caruso (1979), el ser humano se experimenta como modificador de sí mismo y del mundo, pero a su vez, como modificado por el mismo mundo. De ese modo, la técnica psicoanalítica continuamente busca la comprensión de los sujetos desde su complejidad subjetiva e intersubjetiva, es decir, a partir de sus propios procesos internos y de los construidos en interacción con otras personas. Para los estudios psicoanalíticos, esta realidad plantea una importante posibilidad de integración en cuanto a elementos socioculturales. Esto no resulta desatinado si se considera que la formación de los ideales en los individuos y la formación de sus relaciones sociales, solo pueden ser comprendidas en el contexto de relaciones más amplias donde se involucran estructuras históricas y normativas en términos sociales. Por lo tanto, los estudios psicoanalíticos se vuelven sociales cuando le otorgan relevancia y analizan relaciones sociales recíprocas, relaciones que se influyen e interdeterminan, comprendiendo que toda esa estructura repercute en las personas de manera importante, tanto en la construcción de su mente como de su destino. 
Si bien, autores como Bleichmar y Leiberman (1989) advierten sobre la diversidad de escuelas y desarrollos en los estudios psicoanalíticos, en este artículo se realizará un esfuerzo de concentración en elementos básicos y compartidos por las diferentes vertientes en los estudios psicoanalíticos, mismos que a su vez, resulten viables de complementación con los estudios de migración desde una perspectiva sociológica. Con esto se hace referencia a la integración de elementos sociales y subjetivos para la comprensión de dinámicas internas, renunciándose a perspectivas estrictamente clínicas e individuales. No es una problematización reciente la que plantea como necesario que la disciplina psicoanalítica emprenda nuevos caminos metodológicos debido a que la multiplicidad de teorías clínicas contradictorias y la multiplicidad de escuelas psicoterapéuticas, evidencian serias limitantes.

Esta observación es relevante si se recuerda que, en sus inicios, esta disciplina privilegió el informe de caso, lo cual se debió en gran parte a la concentración en actividades clínicas o psicoterapéuticas. Sin embargo, y a pesar de su valía en ámbitos clínicos, la metodología del estudio de caso no es la exclusiva para comprender fenómenos humanos, y mucho menos, para ámbitos sociales (Kachele y Thoma, 2003). Esto no significa que el esfuerzo por relacionar la experiencia migratoria, el funcionamiento mental y la disciplina psicoanalítica no exista previamente, pues autores como Grinberg y Grinberg (1982) plantean un importante precedente.

Por lo tanto, se considera elemental una revisión de antecedentes en cuanto a los estudios sobre migración y salud mental con la intención de rastrear sus perspectivas de estudio, para, posteriormente, revisar los fundamentos epistemológicos de la disciplina sociológica y psicoanalítica respectivamente. Con lo anterior, se plantea llegar a una claridad que permita proponer líneas de estudio con una metodología articulada a través de la cual, se aporte al esclarecimiento del fenómeno migratorio en un sentido interno y subjetivo, integrándose elementos de comprensión para ámbitos mentales y sociales.

\section{Acerca del estudio de salud mental en migración: La perspectiva psicológica, biográfica y narrativa}

De manera tradicional, las investigaciones sobre el fenómeno migratorio han estado sostenidas en explicaciones económicas y sociales. En contraste con esa tendencia, Lee (1966) en su A theory of migration planteaba que la decisión de migrar nunca era completamente racional y que, para muchos individuos, el componente irracional era mucho más determinante, explicándose la migración por emociones pasajeras, enfermedades mentales y acontecimientos accidentales. En coincidencia, Germani (1969) acusó a los estudios de migración de sostenerse sobre motivaciones de índole racional, tales como la económica, sin tener en cuenta la posible complejidad del proceso psicológico que da lugar a la decisión de irse o de quedarse.

Desde entonces, y aun cuando las críticas daban noticia de una serie de pendientes respecto a los estudios de migración, se va encontrando que, en años recientes, el esfuerzo por la vinculación entre el fenómeno migratorio y el ámbito de la salud mental ha rendido frutos. Investigaciones como el modelo de estrés aplicado a la población migrante, estudios epidemiológicos y de aculturación asociados, así como las narrativas de la migración, proponen respuestas al respecto. 
Destacan revisiones como las de Bhugra (2004), quien plantea que el proceso de migración en sí mismo puede estar vinculado con el origen de trastornos mentales. Distingue la vulnerabilidad psicosocial asociada al acto migratorio entendiéndolo como un proceso, siendo que, desde la etapa premigratoria, se pueden rastrear riesgos considerando el tipo de personalidad en los individuos, lo cual está asociado con que la migración resulte traumática para algunas personalidades y para otras no. Respecto al tránsito, considera como posibilidades de desequilibrio la sensación de pérdida, el duelo y el posible estrés postraumático, siendo finalmente que, en el lugar de destino o postmigración, se puede experimentar desequilibrio por el choque cultural y exacerbarse la sensación de pérdida.

En relación, Alvarado (2008) considera que, para comprender la relación que existe entre el proceso de migración y el desarrollo de un problema en salud mental, es necesario tomar en cuenta varias dimensiones, entre ellas, las características de la persona, las condiciones previas a la migración, las características propias del proceso de migración y las condiciones en la nueva sociedad donde se llega a residir.

Derivado de sus investigaciones y desde la franca perspectiva que relaciona a la migración con el desarrollo de una psicopatología, Ingleby (2008) plantea que la más grande evidencia de ello se encuentra en la esquizofrenia. En sus estudios, identificó una alta correlación entre dicha perturbación y la migración. Por su parte, Achotegui (2004) explica que existen estresores y duelos excesivos por los que atraviesan cierto tipo de migrantes, lo cual puede incidir en una reacción en el área mental. Denuncia que, al no considerarse su condición de migrantes, la sintomatología que presentan puede ser erróneamente diagnosticada, provocando que reciban tratamientos inadecuados y que la situación de atención, paradójicamente hablando, se convierta en un nuevo estresor. Bajo esa sintonía, se identifica el trabajo de Nathan Venturini (2006), para quien el cambio de cultura produce una pérdida de identidad y un trauma irreparable. Por lo tanto, y teniendo en cuenta que la migración conlleva un cambio de cultura, la situación sería siempre vivida como catastrófica para el psiquismo porque la cultura de origen es irremplazable. Por esta razón, la población inmigrante portará severas fallas en la transmisión de la identidad a sus descendientes, lo que provoca un trauma transgeneracional. En su opinión, los problemas sociales que recaen en estas poblaciones y sus descendientes son efecto del cambio de cultura.

Por su parte Moro (2004) considera al acontecimiento migratorio como un acto psíquico que lleva al rompimiento del marco cultural interiorizado de los sujetos. De esa manera, y a menudo, emigrar resulta traumático. Sin embargo, y en sus conclusiones, el traumatismo migratorio no es ni constante ni inevitable, pudiendo sobrevenir cualquiera que sea la personalidad anterior del emigrante. En su opinión, los factores sociales hostiles (en el país de origen y en el nuevo país) son factores agravantes. Pero, aun cuando esto suceda, el traumatismo no produce necesariamente efectos patógenos. Algunas veces, como todo traumatismo, puede ser estructurante y portador de una nueva dinámica para el individuo, incluso, puede ser el germen de una transformación o fuente de una nueva creatividad. Por lo tanto, la migración puede ser, también, portadora de potencialidades creadoras. De ahí la necesidad de identificar los factores que permitan controlar el riesgo transcultural (Moro, 2004).

En sintonía con Moro y a diferencia de los autores previos, Vilar y Eibenschutz (2007) concluyen que, probablemente, la migración por sí sola no sea la causa de un deterioro en la salud mental. Sin embargo, consideran que este deterioro puede estar relacionado y detonarse tras complicaciones respecto a la situación de 
empleo, por ejemplo, y acontecimientos traumáticos previos, durante y después de la migración, los cuales, pueden ser motivos suficientes para experimentar una angustia psicológica.

Desde el enfoque sistémico valdría rescatar los trabajos de Falicov (2001) sobre la manera en que las migraciones han contribuido a la emergencia de nuevas formas de familia, esto es, familias trasnacionales, mismas que literalmente viven de un lado y de otro, fragmentadas, sufriendo desventajas, y también ventajas, tanto para aquellos miembros de la familia que se van como para los que se quedan en el país de origen. En consonancia, se encuentran los trabajos de Andolfi (2009) quien se enfoca en el conocimiento del ámbito psicológico en familias pertenecientes a distintos contextos culturales y sociales, profundizando en los desafíos que se deben enfrentar para su mejor atención. Asimismo, este autor señala la necesidad de que los terapeutas revisen sus supuestos acerca de la diversidad y sus propios prejuicios, todo esto, para potenciar sus recursos personales y desarrollar una real capacidad de acercamiento.

Bajo otra línea, autores como Hwang, et al. (2007) se afilian a una corriente que estudia el impacto de la cultura sobre la salud mental, encontrándose, por ejemplo, que la población inmigrante en Estados Unidos ha venido mostrando una carga importante de padecimientos. A nivel de intervención, encuentran una desigualdad en cuanto al acceso de servicios en salud. Plantean que dichos sistemas pueden no estar adecuadamente preparados para satisfacer las necesidades de poblaciones específicas, tales como la migrante. Esto se refiere, además, a una limitada comprensión del factor cultural involucrado en sus padecimientos.

En ese sentido, se cuenta también con investigaciones en el contexto mexicano. En términos epidemiológicos, destaca el estudio de Bojorquez, et al. (2015), quienes se concentraron en la prevalencia de trastornos mentales en migrantes deportados hacia México. En su investigación encontraron porcentajes significativos de personas con necesidad de atención en salud mental. Asimismo, concluyeron que, dicha prevalencia, es más alta en mexicanos migrantes en Estados Unidos, en comparación con los mexicanos en México. A partir de esto, sugieren la consideración de diseños e implementación de políticas públicas en salud. Bajo la misma línea, se encuentra la propuesta de Temores-Alcántara et al. (2015), quienes en un trabajo que tomó como contexto la ciudad fronteriza de Tapachula, Chiapas, exploraron el estado de salud mental y expectativas de atención en migrantes centroamericanos en tránsito. Ellos encontraron que los migrantes presentaban signos y síntomas de daños en su salud mental relacionados con experiencias vividas en el lugar de origen y en el tránsito por México. Concluyeron que es necesario fortalecer la respuesta del sistema de atención en salud mental a partir de estrategias de cooperación y también, de igual manera resaltaban la necesidad de emprender acciones que vayan más allá de una construcción biomédica en salud mental.

Todos los estudios mencionados coinciden en que existe una vulnerabilidad psicosocial específica en la población migrante. Ahora bien, y aun cuando no todos los sujetos migrantes sufren de desajustes en términos de salud mental, es un hecho que sí lo experimenta un porcentaje importante de ellos. Por otro lado, dichos estudios se concentran en la tercera etapa del proceso migratorio. Ello implica restarle importancia a la comprensión de experiencias constructoras de la mente, vivencias que permitirían comprender la necesidad de desplazamiento, las fantasías asociadas, las expectativas depositadas en el acto y su relación con la realidad. 
Desde su hipótesis genética, la perspectiva psicoanalítica comprende los padecimientos y las personalidades actuales de los sujetos con base en una exploración de su pasado remoto. Además, autores como Grinberg y Grinberg (1982), consideran a la migración como una experiencia potencialmente traumática que configura a su vez, una situación de crisis, marcada por dinámicas inconscientes previas, durante y después de la migración. Bajo la misma línea, se podrían identificar propuestas como las de Nathan, Moro y Venturini.

Desde una postura crítica, Venturini (2006) señala la reacción de las sociedades receptoras frente a los movimientos migratorios como de rechazo y de cierre de fronteras, lo que lleva a construir y otorgándosele en general el estatuto de problema al considerarlos como productos de miseria provenientes de otras regiones del mundo. Esto no necesariamente es atinado. Señala que, tanto la psiquiatría como la psicología, particularmente la de los países receptores, se han interesado en analizar las repercusiones psíquicas de estos movimientos poblacionales sin escapar de esta concepción de problema. Esta autora interroga las corrientes que estigmatizan algunos de sus fenómenos señalándolos como trauma o enfermedad, basándose especialmente en la idea de que el ser humano se constituye a partir de una identidad otorgada por la cultura en la que ese sujeto creció. En su conclusión y desde una perspectiva psicoanalítico-lacaniana, sostiene que, determinar que la migración constituye una experiencia traumática, limita el escuchar los modos singulares en que puede tomar forma la emergencia del sujeto, restringe el conocimiento de sus propias interpretaciones sobre la experiencia y la manera en que conforman su identidad. Pensar de esa manera, señala, reduce la complejidad de una dinámica interna y llena de significaciones en los sujetos migrantes.

Como se mencionó anteriormente, la perspectiva biográfica y narrativa en el estudio migratorio ha mantenido también una importante presencia. Se considera relevante puntualizar que el método biográfico permite el estudio y la recolección de documentos personales de vida, historias, recuentos y narrativas que describen momentos de cambio a niveles individuales. Entre ellos, la migración ha fungido como un fenómeno de atención.

A continuación, se destaca el uso y presencia que ha tenido dicho método en los estudios de migración:

1) William I. Thomas y Florian Znaniecki: entre los años de 1919 y 1920 realizaron un estudio sobre campesinos polacos inmigrantes en Estados Unidos, buscando comprender la manera en que transformaban las pautas familiares, sus comportamientos, así como los sistemas de usos y costumbres. Para Blumer (referido en Ariza y Velasco, 2015), este es el primer estudio sobre migración que plantea el estudio de la subjetividad en población migrante (Ariza y Velasco, 2015).

2) Manuel Gamio: su obra es considerada como la segunda más importante respecto a la subjetividad en población migrante, aunque menos conocida, inclusive entre migrólogos mexicanos. Su estudio The mexican inmigrant: his life story, es el resultado de la investigación sobre la inmigración mexicana a Estados Unidos. Presenta una serie de entrevistas en forma de conversaciones realizadas entre 1926 y 1927 (Ariza y Velasco, 2015).

3) Ricardo Pozas: Juan Pérez Jolote muestra el rostro indígena jornalero del suroeste mexicano. Considera el relato de vida en primera persona de un indio tzotzil, quien narra una parte de su vida en desplazamiento desde la localidad de 
Chamula a los pueblos cercanos, y luego como trabajador en la ciudad de San Cristóbal, como jornalero en el Soconusco y también como soldado por el centro del país en plena revolución mexicana. El sujeto, a su regreso al pueblo natal, enfrenta la falta de prestigio para alguien que no creció en el pueblo, por lo que realiza esfuerzos por demostrar que merece ser aceptado como parte de ella. Toca pues el tema de la relación entre prestigio y migración o redes migrantes y subordinación étnica (Ariza y Velasco, 2015).

4) Oscar Lewis: la segunda obra pionera de este autor, Los hijos de Sánchez, estudia el proceso de proletarización de los campesinos inmigrantes a la Ciudad de México a fines de la década de los años cincuenta, en pleno proceso de industrialización y urbanización del país. Se analiza la transformación de la familia como consecuencia de la migración campo-ciudad y ejemplifica también su concepto de cultura de la pobreza (Ariza y Velasco, 2015).

5) Jorge Bustamante: se refiere a la utilización de lo que el autor llama método autobiográfico en el cual realiza una serie de entrevistas a un emigrante mexicano en Estados Unidos; dicho trabajo tuvo por título Don Chano. Autobiografía de un emigrante mexicano, publicado en 1971 (Bustamante, 1997).

6) Marilyn P. Davis: Mexican voices/American dreams se publica en 1990, documentando la migración de la zona central de México a Estados Unidos usando 90 relatos de vida (Ariza y Velasco, 2015).

7) Ruth Behar: en 1993 se publica Translated women.Crossing the border with Esperanza's store, donde se relata la historia de una mujer migrante de una localidad rural de México. En este trabajo se analizan las condiciones de dominación y violencia, así como el deseo de llegar a Estados Unidos por parte de la sujeto de estudio (Ariza y Velasco, 2015).

8) Jorge Durand: destaca su trabajo de $1996 \mathrm{El}$ norte es como el mar. Su fundamento, fue entrevistas a trabajadores migrantes en Estados Unidos. Buscó reconstruir la historia migratoria de trabajadores mexicanos que laboran en el país del norte (Ariza y Velasco, 2015).

9) Federico Besserer: en 1999 es publicado Moisés Cruz: historia de un transmigrante, investigación que muestra la no linealidad del proceso migratorio y la simultaneidad de los acontecimientos ligados a los mercados de trabajo agrícolas. Utiliza el método biográfico para cruzar historias de migrantes activistas de origen mixteco en Estados Unidos y con ello dar cuenta de la formación de organizaciones locales y étnicas transnacionales (Ariza y Velasco, 2015).

10) Lynn Stephen: de manera más cercana en términos temporales, se identifica el trabajo titulado Transborder lives. Indigenous Oaxacans in Mexico, California and Oregon, de 2007, en el que se da cuenta de cómo la experiencia de la frontera geopolítica va más allá del lugar físico. Esto resulta al estudiar a través del relato de vida, los sufrimientos de diversas personas de origen indígena en Oaxaca y en el estado de Oregón, en Estados Unidos (Ariza y Velasco, 2015).

11) Abdelmalek Sayad: analiza las vivencias de los inmigrantes argelinos en Francia en relación con las condiciones históricas, sociales y económicas de las sociedades expulsoras y el manejo sociopolítico de la migración en las sociedades acogedoras. La doble ausencia. De las ilusiones del emigrado, a los padecimientos del inmigrado destaca entre sus trabajos más relevantes (Ferrant, 2018).

12) Matilde María Criado: en La línea quebrada, historias de vida de migrantes, analiza el fenómeno migratorio a partir de los testimonios y trayectorias de vida 
de emigrantes extranjeros radicados en Madrid y con origen en las distintas áreas geográficas. Incluye la reconstrucción de un número significativo de historias de vida que ilustran y fundamentan el análisis sobre los condicionantes e implicaciones que acompañan a este proceso (Criado, 1999).

13) Leonor Arfuch: profundiza respecto al tema de la identidad asociada a la intensificación de los tránsitos migratorios. Entre sus obras destacan La vida narrada. Memoria, subjetividad y política (Arfuch, 2005).

14) Juan F. Marsal: a comienzos de los años cincuenta, publica su obra Hacer la América, la historia de vida de un emigrante español. En paralelo, deja un legado sociológico relevante, teórico y metodológico. El método de los testimonios, de los investigados y del investigador encierra una propuesta tradicional e innovadora a la vez de oficio sociológico (Valles, 2009).

Todos estos trabajos biográficos y narrativos coinciden en que, siguiendo un principio paradigmático común, comparten la escucha como la principal actitud del investigador, la perspectiva de un mundo vital autocontenido lo cual supone que la historia o relato de vida ha sido construido por los mismos sujetos. De igual forma, la empatía controlada, entendida como el acto de comprensión hacia la persona, así como una familiarización con su contexto de significados y su manera particular de interpretar su realidad. Finalmente, comparten la proyección de tiempos, estimulándose la reconstrucción episódica del pasado, así como la proyección de deseos en futuro. En conclusión, estas investigaciones comprenden que lo relatado no necesariamente corresponde a la realidad, sin embargo y para ellos, la fidelidad no es lo sustancial, sino la manera en que los sujetos reconstruyen y reinterpretan los significados simbólicos de sus experiencias específicas. Todo esto tiene una amplia coincidencia con la perspectiva psicoanalítica. A continuación, se revisará lo correspondiente a sus cruces epistemológicos con la disciplina sociológica.

\section{Las tradiciones metodológicas en sociología}

En cuanto a sus orígenes, las tradiciones metodológicas en sociología obedecen a un contexto específico, la Europa de finales del siglo xix y principios del xx. En Francia destaca la obra de Emilio Durkheim y en Alemania, el proyecto de Max Weber. Los dos autores representan corrientes teóricas y metodológicas diferentes, pero a su vez, ofrecen las bases desde donde se desarrollan las tradiciones que adoptan las comunidades de científicos sociales desde entonces (Tarrés, 2013). Es importante puntualizar que actualmente, la sociología es una disciplina que se trabaja desde enfoques y teorías diversas que le complejizan y le llevan más allá de un saber monolítico. Sin embargo, y al igual que en el caso de la disciplina psicoanalítica, se opta por una revisión desde sus fundamentos clásicos que aún mantienen vigencia.

En Francia, Durkheim argumentó que los fenómenos sociales son cosas y deben ser tratados como tales en el estudio sociológico. Con cosa se refiere a todo lo que está dado y se impone a la observación. Por lo tanto, tratar a los fenómenos como cosas, es tratarlos en calidad de data que constituyen el punto de partida de la ciencia (Durkheim, 1895). Por lo tanto, la tarea de la sociología que propone, es el estudio de los hechos sociales, concibiéndolos como "las formas de actuar, pensar y sentir, exteriores al individuo, dotados de un poder de coerción, gracias al cual se imponen" 
(Tarrés, 2013). En su opinión, la primera tarea del sociólogo es definir las cosas de las que trata. Debe expresar los fenómenos en función, no desde una idea del espíritu, sino de propiedades que le son inherentes, es decir, elementos integrantes de su naturaleza, exteriores, no por su conformidad con una noción más o menos ideal. Planteaba que la actitud del sociólogo debía ser similar a la del científico natural, a quien por regla se le exige que se aparte de datos sensibles que pueden ser demasiado personales, para, en cambio, retener exclusivamente los que presentan un grado suficiente de objetividad (Durkheim, 1895). De manera coincidente, este ideal que separa al investigador y le exenta de todo efecto en la investigación, también resultó ser el ideal en los primigenios estudios psicoanalíticos freudianos.

Freud (1910) descubrió que la labor del psicoanalista es interferida por un fenómeno parecido al que experimenta el paciente. Consideraba que también en él se reactivaban elementos de su propio funcionamiento psíquico, surgiendo impulsos y sentimientos hacia el paciente, ajenos a su función de comprender e interpretar las resistencias y los complejos infantiles de quien solicitaba su apoyo. Llamó a este fenómeno contratransferencia y autores como Racker (1986), señalan que, en el inicio de la técnica psicoanalítica, este fenómeno fue considerado como una perturbación y un serio peligro para la labor del analista, por lo que debía ser evitada en rescate de un ideal de objetividad. Sin duda, esto es similar al ideal de objetividad en los estudios sociológicos de Durkheim.

Vale destacar que, posteriormente, el mismo Freud y estudiosos posteriores como Racker (1986) y Heimann (1950), redefinieron el fenómeno de la contratransferencia como un instrumento muy importante para la comprensión de los pacientes y para la función de intérprete del psicoanalista. Posteriormente, Freud mismo consideró que la manera en que se podría evitar que entorpeciera los procesos inconscientes del análisis, debía partir a través de un esfuerzo por parte del psicoanalista, exigiéndosele una capacidad para tomar noticia de sus propios complejos; esto se obtenía a través del análisis propio o llamado análisis didáctico. Esto se refiere a que la contratransferencia es una reacción ineludible que interviene en la manera de ser y en la conducta del psicoanalista, que paradójicamente, puede ponerse a disposición de la comprensión del paciente, donde a partir de una situación objetiva como lo es el encuentro analítico, la persona podría encontrar comprensiones mayores a las que ha tenido sobre su realidad o fantasías a partir del uso de la contratransferencia.

Volviendo a Durkheim, él considera que el método de investigación indicado es el comparativo, pues posibilita la contrastación de un mismo hecho social en sociedades y épocas similares o distintas. La comparación así planteada, es un equivalente a la experimentación en ciencias naturales (Tarrés, 2013). Sin embargo y a pesar de este aparente rígido posicionamiento metodológico, Durkheim fue un investigador que utilizó información cualitativa en sus investigaciones, lo cual es identificable en su estudio sobre el suicidio (Durkheim, 1897). Resulta, por lo tanto, evidente que su método y teoría aceptan cortes cuantitativos y cualitativos de la realidad que investiga. En sus palabras "el sociólogo puede recurrir a la historia, a las observaciones de los textos antropológicos o a las estadísticas para construir su objeto" (en Tarrés 2013, p. 47).

La segunda tradición importante en sociología se orienta a la comprensión de los significados de la acción y de las relaciones sociales. Este paradigma critica el supuesto positivo de que los hechos sociales son únicamente objetivos. Para Weber (1922), el fin de la sociología es lograr una comprensión de los significados subjetivos de la acción pero que tienen vinculación con motivos racionales que pueden ser identificables 
empíricamente, lo cual permite explicar las causas de la acción. En ese sentido, propone la concepción de leyes bajo condiciones muy específicas y diferentes a las planteadas por las ciencias naturales. Se refiere a la identificación de probabilidades típicas, confirmadas por la observación, que han sido comprensibles por sus motivos comunes, por el sentido mentado por los sujetos de acción y por un esfuerzo intelectual de quien le estudia. De esta manera, se vuelven claras y comprensibles en un sentido racional con arreglo a sus fines. Es así como en Weber se identifica una crítica hacia los prejuicios naturalistas, pues, hasta ese momento, todo lo que se llamase científico debiera ser un símil de las ciencias naturales exactas, una extrapolación directa. Sin embargo, en sociología, esto había llevado a una suerte de automatización con la consecuente incomprensión del sentido de las formaciones teóricas estudiadas (Weber, 1958).

Por lo tanto, la sociología construye conceptos-tipo, y se afana por encontrar reglas generales del acaecer. Esto, señala, es alcanzable a través de conceptos y reglas racionales. Sin embargo, continua Weber (1922), la sociología busca también, aprehender mediante conceptos teóricos y adecuados por su sentido, fenómenos irracionales. Esto es útil para la sociología en la medida en que, mediante la indicación del grado de aproximación de los fenómenos, quedan ordenados conceptualmente. Por lo tanto, en el dominio de su propuesta sociológica, solo se pueden construir "promedios" y "tipos-promedio" que, siendo plurales, convergen en una misma razón.

De esta manera, la tarea de la sociología es más limitada pero también más compleja. Se debe orientar a establecer generalizaciones capaces de definir tendencias sobre la naturaleza, el curso y las consecuencias del comportamiento social. Ello es posible porque el comportamiento social tiende a presentar regularidades, a seguir patrones que se repiten. Sin embargo, no basta expresar una tendencia en términos cuantitativos o estadísticos. Es preciso fundamentarla en una interpretación comprensiva del comportamiento para que se pueda entender su significado.

Ligado a esto, conviene agregar que para Weber (1922), toda interpretación persigue la evidencia, pero ninguna interpretación de sentido, por evidente que sea, puede pretender solo por su carácter de evidente una interpretación causal válida. Este tipo de realidades promueven más bien hipótesis causales particularmente evidentes que, con frecuencia, encubren motivos que no son claros inclusive para el mismo actor social. Encierra una conexión real, una trama en su acción, de manera que el propio testimonio subjetivo, aunque sea sincero y a consciencia, solo tiene valor de relativo. En este caso, la tarea de la sociología es averiguar e interpretar esas conexiones, aunque no hayan sido elevadas a la conciencia. Se buscaría la comprensión de impulsos contrarios, aunque la deducción no resulte de total seguridad. Serán, por lo tanto, sus resultados, sus acciones o la realidad misma del curso del fenómeno, lo que ilustre sobre lo atinada o desatinada que fue la comprensión que se presumió sobre ellos.

Integrando a ambos autores para los fines de este artículo, se rescatan algunos aspectos. En primer lugar, que más allá de la tajante dicotomía entre explicación y comprensión, los debates metodológicos no pueden separarse de las tradiciones teóricas y sus fundamentos epistemológicos. Es clave el hacer coincidente el objeto de estudio con el fundamento teórico en que se sustentará la aproximación, por lo tanto, el conocer el fundamento epistemológico de tal o cual corriente, posibilita el saber los alcances que se tienen a través del estudio y lo que este exigirá del investigador, ya sea una observación y análisis detallado del fenómeno a través de la explicación, o una aproximación empática a través del ejercicio de la comprensión y búsqueda 
de significados. En segundo lugar, que, aunque el ideal de investigación exija el posicionamiento en una postura epistemológica, se ha identificado que, en ambos autores, este purismo no resultó posible, siendo ambos fundamentos intercalados en sus exploraciones, lo cual se debe a la complejidad del estudio social. Por lo tanto, las metodologías mixtas se consideran relevantes al realizar estudios sociales.

Autores como Creswell (2009), consideran que los métodos mixtos se caracterizan por retomar, en una sola línea de investigación, las fortalezas tanto de la metodología cualitativa como de la cuantitativa, además, resultan viables de asociación en áreas específicas del conocimiento tales como las investigaciones en ciencias sociales y en ciencias de la salud, resultando convenientes para abordar la complejidad de esos fenómenos. Asimismo, permiten una mayor profundización en los análisis en comparación de estudios aislados. En los apartados posteriores, se brindará un panorama sobre esta realidad en los estudios psicoanalíticos, los cuales, también han atravesado por los dilemas epistemológicos de las tradiciones cuantitativas y cualitativas, pero con importantes puntos de cruce que les vuelven coincidentes con los estudios en ciencias sociales.

\section{Los fundamentos de la teoría psicoanalítica: Una discusión sobre sus orígenes epistemológicos}

Quizás de manera sorprendente para algunos, en la primera etapa de construcción de la teoría psicoanalítica se optó por un monismo epistémico y una adherencia a postulados fisicalistas según los cuales, únicamente las fuerzas químicas y físicas actúan en el organismo. Será así que, por algunos años, la metapsicología fundada por el psicoanálisis y sostenida en gran medida desde el positivismo, configurará su identidad epistémica (Herrera, 2013).

En esos momentos, la línea de abordaje fue la tópica, es decir, la concepción hipotética de una anatomía psíquica que iba en un rodeo de la medicina a la neurología. Pero esta propuesta empírica se basaba en un razonamiento específico, este era que existe una anatomía psíquica, pero también, psicopatologías como la histeria que se comportan como si dicha anatomía no existiera. Con esto se hacía alusión a la existencia de procesos y mecanismos inconscientes con funcionamientos propios y específicos. Funcionamientos complejos y que, en algunos casos, resultaban contradictorios con lo ya conocido, desafiando los descubrimientos que en el siglo xix se habían obtenido a través de la psicología experimental y la neurología. El naciente estudio se enfrentaba a un problema de delimitación epistemológica, debido a que los datos registrados contradecían la expectativa anatomo-patológica. Es importante recordar que Sigmund Freud fue formado en una tradición donde la fisiología era considerada como una continuación de la física, siendo que la energía era la sustancia que unía a dos campos disímiles hasta entonces, la neurología y la psicología (Herrera, 2013).

La en ese entonces, naciente propuesta psicoanalítica, requeriría de esfuerzos que le definieran desde su especificidad y le diferenciaran respecto a los saberes dominantes de esa época. En consonancia con esto, se considera relevante destacar un importante distintivo epistemológico en el saber propiamente psicoanalítico que viene a distinguirle de otros enfoques asociados a lo mental. Es bien sabido que, por ejemplo, en los estudios neurológicos y médicos, prima la mirada. A diferencia 
de esto, en psicoanálisis se privilegia la escucha como vía de acceso primordial a lo inconsciente y, por tanto, al funcionamiento del aparato psíquico (Herrera, 2013). Esto es un elemento clave que ha distinguido al estudio psicoanalítico, pero a su vez, que le vuelve coincidente con estudios sociales basados en métodos biográficos y narrativos, que privilegian la escucha y análisis de discursos para la comprensión de las personas migrantes. Esto es claro al retomar posturas como la de Weber que busca los significados de la interacción y que se sostiene a partir de la escucha para el análisis y emisión de conclusiones. Tomando este ejemplo, se identifica una importante coincidencia con la metodología psicoanalítica.

Por lo tanto, los métodos sociológicos que otorgan una importancia primordial a la escucha, a la búsqueda de significados y a la consideración de la realidad de los mismos sujetos como valiosa, son coincidentes. Se hace referencia a estudios que realizan un esfuerzo por humanizar (contrario a la predilección del dato duro y estadístico) la experiencia en sujetos como los migrantes, es ahí donde se presenta un cruce, un intercambio viable con los estudios psicoanalíticos.

El esfuerzo psicoanalítico es claro, el síntoma designa algo más que la sola disfunción. Es evidente que todo síntoma se revela por una falla en el funcionamiento normal, pero el síntoma en sí, es únicamente la puerta de acceso y no el fin, ya que más bien, está vinculado con la emergencia de una verdad. Por lo tanto, la eliminación del síntoma no es lo primordial en la comprensión e intervención psicoanalítica, sino que, interpretarlo, equivale a descifrar la verdad que contiene. Se entiende pues al síntoma como una alteración que puede ser comprendida dentro del amplio espectro de estrategias subjetivas de defensa (represión, negación, racionalización, escisión, etc.). La anomalía, por lo tanto, no es en sí patológica, la patología es denominada por el experto en salud. En psicoanálisis se entiende que el síntoma expresa otras posibles normas de vida. Conforme el tránsito en su delimitación epistemológica, los estudios psicoanalíticos van distinguiéndose como disciplina separada a lo concebido en esa época, inclusive, aun de las llamadas psicologías de la conciencia (Herrera, 2013).

Recapitulando, un inicio positivista en psicoanálisis es innegable, sin embargo, se identifica también y gradualmente, una separación respecto a las búsquedas de medición, prefiriendo la escucha que llevaría a la cualidad y a la comprensión, entendiéndola como mediada por la conciencia y, por tanto, incierta. Con esto se enfatiza que cuando se mide a partir de la consciencia, se mide la intensidad que en la conciencia alcanza un hecho psíquico, y solo eso, pues persiste el desconocimiento sobre la cualidad del hecho en sí, cualidad que será buscada por la metodología psicoanalítica. En síntesis, los estudios psicoanalíticos presentan una línea epistemológica que no es posible encasillar estrictamente en el terreno de lo positivista ni estrictamente en lo humanista pues rescata de ambos. A propósito de las evidencias necesarias en las comunidades científicas, Shedler (2010) señala que pocos psicoanalistas se dedican a la investigación por lo que, a pesar de la acumulación de evidencia empírica en los estudios psicoanalíticos, no se suele respaldar la práctica con esto. Para este autor, es importante integrar al investigador psicoanalítico, aun con lo desconocido que resulte el manejo estadístico en este tipo de métodos, es decir, estudios con base empírica o que rescaten de las metodologías cuantitativas algunas aproximaciones a fenómenos que involucren lo humano. Esta reflexión coincide con la solidez metodológica buscada desde la sociología, siendo una posibilidad adicional de diálogo entre ambas disciplinas, pero en específico, con los estudios de migración que, como se vio al inicio del texto, presentan una larga tradición cuantitativa u objetiva, a través de la cual, los 
estudios psicoanalíticos pudieran aproximarse a una comprensión del fenómeno con base en estudios de larga data.

Perrés (1988) se encargará de poner en duda, con base en una revisión histórica profunda de los desarrollos teóricos en psicoanálisis, su cualidad tanto positivista como humanista, entendiendo que cuando se le estudia, es conveniente integrar todos los elementos que le construyen y hacer honor a su objeto de estudio, esto es, que es clave integrar el elemento inconsciente al hablar de epistemología psicoanalítica.

Un hecho sorprendente, revela Herrera (2013), es que, si se lleva a cabo la tarea de revisar cuidadosamente la obra completa de Freud, uno no encontrará referida ni una sola vez la palabra epistemología, siendo la salvedad, una carta enviada a Carl Jung, donde refiere: "me está rondando un trabajo acerca de la dificultad epistemológica del inconsciente, para lo cual me llevaré unos libros este verano" (carta a Jung del 1ro. de julio de 1907, en Herrera, 2013, p. 176). Para Perrés (1988), este hecho es clave y no siempre tomado en consideración por los teóricos en psicoanálisis. La ausencia de profundas reflexiones epistemológicas en psicoanálisis devela una evasión importante a revisar.

Se considera no poco importante, señalar también que existe una falta de acuerdo respecto a las bases epistemológicas en psicoanálisis, lo que llevaría a un posicionamiento en relación con sus estudios con fundamento positivista, los filosóficos humanistas althuserianos y los que reclaman una epistemología específica. Autores como Otto Fenichel, Franz Alexander, Karl Meninger, Santiago Ramírez y Miguel Kolteniuk encabezarán los esfuerzos del positivismo en psicoanálisis, sin dejar de lado a los psicólogos del yo (Perrés, 1988).

Fue la escuela generada por el filósofo Louis Althusser la que provocó un importante interés por los problemas epistemológicos de las diferentes disciplinas científicas, entre ellas, el psicoanálisis. Para este autor, el psicoanálisis posee claramente un objeto de estudio: el inconsciente y sus efectos, y, para su conocimiento, está dotado de una teoría y una técnica (método). Esto llevaría a reconocer el descubrimiento de Freud, dotarlo de conceptos teóricos adecuados de manera rigurosa, estos son, el inconsciente y sus leyes. Para Althusser, la propuesta de Freud posee la estructura de una ciencia, conteniendo: a) una práctica (la cura analítica), b) una técnica (el método de la cura y, c) una teoría que está en relación con la práctica y la técnica. De esa manera señala que, dicho conjunto, orgánico-práctico, técnico y teórico, remite a la estructura de toda disciplina científica (Althusser, 1965).

Perrés (1988) vuelve más compleja la articulación de fundamentos para el conocimiento psicoanalítico pues plantea que su sostén no puede reducirse a su producción teórica sino a una compleja articulación entre ella, la clínica y la dimensión del análisis propio (refiriéndose a la de los mismos analistas). Tomando las reflexiones de Mannoni, considera que el estatuto científico del psicoanálisis no únicamente se vincula por la teorización (línea althuseriana), ni por los descubrimientos empíricosclínicos con sus pacientes (línea positivista), sino por la articulación de esos aspectos con el movimiento interno en relación con la dinámica de transferencia. Antes de profundizar en esa dinámica, vale reafirmar que la dicotomía explicar-comprender, no es tan radical en psicoanálisis (al igual que en la sociología de Durkheim y Weber) siendo que, desde Freud, la explicación natural y la comprensión hermenéutica se suceden, además de que, la reflexión epistemológica propia del psicoanálisis no puede quedarse sólo con el discurso teórico o con el discurso clínico, sino que debe poder hablar desde ambos, mostrando permanentemente sus articulaciones. 
Volviendo al tema de la transferencia del analista (técnicamente llamada contratransferencia y que se mencionó anteriormente), podría ponerse de ejemplo que un analista entiende la teoría de una manera cuando únicamente le conoce desde ahí, a diferencia de cuando lo hace bajo la experiencia del propio análisis. Por lo tanto, será importante diferenciar dos discursos en psicoanálisis, el teórico y el clínico, los cuales no son directamente homologables y que más bien, mantienen sus propios registros de funcionamiento.

Volviendo al tema de la transferencia, Perrés (1988) planteará que el investigador y clínico en psicoanálisis deberá tener cuidado con la capacidad protectora de la teoría. Por ejemplo, valdría la ejemplificación del psiquiatra cuando se ubica como el "sano" frente al paciente para no caer en angustias transferenciales, refugiándose en su función, aferrándose a la protección de la teoría. Por lo tanto, si el psicoanálisis se presenta como disciplina y asumiendo sus particularidades que la hacen diferente a otras disciplinas, su epistemología debiera integrar esas particularidades en vez de descartarlas, de otro modo sería trágicamente incongruente.

Dichos elementos de integración son convenientes para un alcance mayor en la complementación con los estudios de migración, pues la manera de comprender al sujeto migrante implicaría que el investigador se conciba como un sujeto involucrado en la dinámica y en reacción al discurso y presencia de la persona migrante, elementos que una vez integrados al estudio, pudieran aportar comprensiones mayores.

Siguiendo lo anterior, la idea de concebir el psicoanálisis como una teoría hermenéutica, resultaría en otro reduccionismo. Sin duda, esta línea está presente y es sumamente valiosa, pero no es la única, ya que la dimensión de la comprensión (hermenéutica) también aparece emparejada con la explicación en psicoanálisis. Por lo tanto, otro aspecto de su especificidad radica en la unión de los dos ámbitos básicos claves de la historia científica: el comprender y el explicar.

Se considera clave retomar a Devereux (1967), quien como investigador social con formación psicoanalítica, identificó la dinámica de la transferencia en los procesos de investigación ligándola a conceptos como angustia y su papel en la generación del conocimiento. Desde esta perspectiva, el investigador no está libre de la contratransferencia, siendo esta la suma total de las distorsiones en la percepción que tiene de su objeto de estudio, y la reacción ante él que le hace responder como si fuera una imagen temprana, y obrar en la situación en función de sus necesidades, deseos y fantasías por lo general inconscientes.

Para este autor, a la pregunta ¿cuál es el camino para la claridad y redireccionamiento de la angustia en la investigación?, le seguiría la siguiente respuesta: a través del trato de las reacciones del científico respecto a su material y su trabajo como el más fundamental de todos los datos de esa ciencia, de lo contrario, solo se tendrá la ilusión de la simplicidad. Su autenticidad aparecerá cuando se comprenda la necesidad de que los hombres que actúan en el desarrollo del conocimiento, sean personas perfectamente conscientes de su propia humanidad, precisamente, cuando más plenamente la pongan en obra en su labor científica, entendiendo que toda investigación es siempre -en forma profunda- una investigación sobre el investigador mismo que remite a sus motivaciones inconscientes (Devereux, 1967). Ligado a esto y no como tema menor, resulta conveniente poner sobre la mesa el tema del narcisismo del investigador, lo que acercaría a una posible explicación respecto a los puntos ciegos en los estudios que, entre otras cosas, podrían estar relacionados con el poder 
tolerar la herida narcisista que significaría para el estudioso, por ejemplo, la caída de su hipótesis de trabajo, sus desarrollos teóricos o la falta de acuerdos en comunidades científicas.

Autores como Kachele y Thoma (2003), a pesar de dirigir sus esfuerzos a la investigación psicoanalítica en el plano clínico, se consideran convenientes para afinar lo que se va comprendiendo como especificidad epistemológica del psicoanálisis y como eje guía, su vinculación con los estudios de migración. Estos autores reivindicarán el tema de la hermenéutica como sustento para la clínica psicoanalítica, pero desde revisiones más profundas.

Para ellos, la actividad hermenéutica entendida como ejercicio de comprensión en la investigación resulta viable de vinculación con la psicología comprensiva, refiriéndose a la exigencia de empatizar o ponerse en lugar del otro. Esa exigencia posibilita el proceso psicoterapéutico psicoanalítico. Introspección y empatía son características esenciales de las reglas técnicas en psicoanálisis. Para Kohut, Freud tornó utilizables la introspección y la empatía como instrumentos científicos para la observación sistemática y el descubrimiento (Kachele y Thoma, 2003). La guía para esto tiene que ver con que el psicoanalista accede a conductas actuales que son incomprensibles en un paciente, pero que se vuelven con sentido a través de la persecución de su desarrollo, teniendo lugar la comprensión histórica-genética, la comprensión de fenómenos psicológicos y/o patológicos en su nexo más estrecho con su biografía (elemento clave en la investigación con población migrante con base en el método biográfico).

En la postura de Ricoeur existe un problema de ilusión al interpretar la consciencia. Para él, esta puede ser engañosa, lo que llevaría a tomar con cuidado la fenomenología que le considera como la totalidad a estudiar y a comprender. Para este autor, el problema de la consciencia es tan oscuro como el problema del inconsciente. Para librar estos problemas se requeriría de una doble confesión: "no comprendo el inconsciente a partir de lo que sé de la consciencia, incluso del preconsciente, pero tampoco comprendo lo que es la consciencia” (Ricoeur, 1969, p. 95).

Por lo tanto, la comprensión de lo inconsciente, al igual que de la consciencia, no es ni completa ni directa. Ricoeur (1969) planteará, a partir de la propuesta freudiana, que lo profundo o pulsional solo se manifiesta a través de representaciones. Se refiere al realismo empírico cuando afirma que lo inconsciente es cognoscible por sus "representantes-representativos" de la pulsión, mismos que son del orden del significado, por lo tanto, coincidentes con el ámbito del habla.

Por lo tanto y siguiendo a Ricoeur (1969), el psicoanálisis se concentra, estrictamente hablando, en el estudio de los derivados del inconsciente y no del inconsciente en sí, por lo tanto, es válido afirmar que el inconsciente tiene una existencia real como la del objeto físico, y al mismo tiempo, que solo existe (o es aprehendido) en lo relativo a sus "derivados" que lo hacen aparecer en el campo de la consciencia. Para este autor, es al afirmar lo anterior cuando nos enfrentamos a una nueva epistemología, una nueva línea de conocimiento que puede ser descubierta a partir de una hermenéutica específica relativa a las reglas mismas del análisis. Es pues, en este análisis o setting, que surge la dinámica intersubjetiva donde los análisis referentes al inconsciente son significantes para el otro (analista) y pueden ser descifrados. En este sentido, se habla de una segunda consciencia en la relación terapéutica, sin embargo, el inconsciente es elaborado esencialmente por un otro (analista), en tanto objeto de una hermenéutica 
que la conciencia propia no puede hacer sola. La consciencia testigo del inconsciente no mantiene solo una relación terapéutica sino también de diagnóstico. En sus palabras dirá sobre la relación terapéutica de desciframiento en psicoanálisis:

Nos limitamos generalmente a definir el inconsciente en relación con la consciencia que lo "contiene". El papel de la otra consciencia no se considera esencial, sino accidental, reducido a la relación terapéutica. Sin embargo, el inconsciente es elaborado esencialmente por otro, en tanto objeto de una hermenéutica que la conciencia propia no puede hacer sola (Ricoeur, 1969, p. 101).

Y añade:

Diremos, pues, que el inconsciente es un objeto, en el sentido de que está "constituido" por el conjunto de operaciones hermenéuticas que lo descifran. No existe de manera absoluta, sino relativa a la hermenéutica como método y como diálogo. Por eso, no se debe ver en el inconsciente una realidad fantástica que tiene el extraordinario poder de pensar en mi lugar" (Ricoeur, 1969, p. 101).

Kachele y Thoma (2003) afirman que fue a través del supuesto del inconsciente que las reglas de interpretación filológicas e históricas adquirieron una dimensión más profunda, siendo que podría llamarse como "hermenéutica profunda" a la técnica interpretativa en psicoanálisis, tal como la plantean Habermas y Lorenzer. La experiencia de conocimiento en la clínica psicoanalítica surge, por lo tanto, desde el primer encuentro entre investigador e investigado, en el sistema técnico que pone a disposición la posibilidad para que surja un lenguaje especializado que tiene como base una teoría y técnica, a partir del cual, se buscan conexiones causales que permitan la comprensión de conductas que, sin este referente, resultarían inentendibles.

Kachele y Thoma (2003) consideran de una valía imprescindible lo concerniente a la intuición como origen de las interpretaciones. Sin embargo, esta revisión científica no acaba ahí puesto que, es totalmente válido un escepticismo respecto de una intuición que cree poder trabajar sin reasegurarse retroactivamente sobre la base de datos objetivos y sin someterse continuamente a verificación. Esto resulta coincidente con la postura de Weber en el sentido de la evidencia sistematizada como sostén. Para estos autores, no resulta completo reducir lo psicoanalítico a una línea epistemológica tajante, o de explicación o de comprensión, sino que es una articulación.

Se podría decir, que Freud objetivó los fenómenos descubiertos e interpretados en las sesiones de psicoterapia mediante una descripción controlable, y los puso en conexión causal e histórico-genética, interpretando por supuesto, pero no limitándose a ello, sino formulando teorías explicativas que probaron ser correctas en diversa medida (Kachele y Thoma, 2003).

A manera de reflexión final, se retoma la postura de Allport quien caracteriza a la labor científica como el intento de "comprender, prever y controlar" un fenómeno específico. Se retoma, debido a que frecuentemente se desvaloriza el rol de la comprensión en la generación del conocimiento y se le emparenta con especulaciones filosóficas y reflexivas, con lo cual se descuida que, el principio hermenéutico, es precondición de cualquier otro paso del quehacer científico, así como también, que la misma labor de comprensión en el ejercicio académico, se fundamenta y sistematiza a partir de un método (Kachele y Thoma, 2003). En relación con esto, vale rescatar que la complejidad de los estudios psicoanalíticos radica en la articulación de las líneas 
de explicación y comprensión a nivel del objeto de estudio, sin embargo, su tarea no termina ahí pues también integra a la figura del investigador o del psicoanalista como sujetos que experimentan reacciones psíquicas inconscientes, lo cual, se relaciona con la manera de interpretar y explicar tal o cual fenómeno.

\section{Una propuesta de ruta para la complementariedad entre ambas disciplinas}

A lo largo del artículo se han identificado importantes puntos de contacto entre los estudios psicoanalíticos y los de migración con fundamento en la sociología, puntos que van desde la problematización y necesidad de estudios que enfaticen en las experiencias subjetivas de los sujetos migrantes, así como en la humanización del mismo proceso migratorio. Dentro de la exploración, también se identificaron fundamentos epistemológicos coincidentes. A continuación, se desarrolla brevemente lo que se propone en cuanto a un estudio que considere la posibilidad de complementariedad entre ambas disciplinas, es decir, la unión de dos discursos disciplinarios que respeta la autonomía de cada uno de sus paradigmas y discursos teóricos, estructurándoles en una convergencia epistémica. Se propone:

a) Una exploración de elementos psicosociales constructores de la mente en los sujetos migrantes, los cuales, son viables de rastreo a partir de sus propias narrativas y con base en una metodología dirigida a la escucha sostenida en un cuerpo teórico, tal es el caso del enfoque psicoanalítico. Se considera que estas exploraciones podrían ayudar a comprender las motivaciones psicológicas para la migración, significados asociados, su impacto y el manejo de la misma experiencia. Con esto no se busca repetir el énfasis en el momento de adaptación exclusiva en el lugar de destino, sino más bien, generar una exploración a través de la cual puedan identificarse significados, impactos psicosociales y funcionamiento mental de los sujetos migrantes. Esta afirmación se sostiene en los métodos biográficos y narrativos, que, a partir de una escucha empática, buscan comprender el desarrollo de fenómenos determinados en los sujetos, en cuyo lugar podría considerarse lo psicológico, pero sin dejar de lado el análisis sociológico, el cual comprende que dicha construcción fue nutrida por experiencias sociales de vinculación asociadas a la migración. Esto es, que llegaron a significarle por un intercambio entre procesos mentales internos e inconscientes, en cruce con dinámicas socioculturales de impacto en los mismos ambientes de desarrollo.

b) Desarrollar, sobre todo, este tipo de perspectivas en los contextos fronterizos, los cuales exigen investigaciones que se concentren en aspectos no solamente ligados a la cuantificación del fenómeno, sino de una comprensión a su vez general, como particular y compleja. Propuestas como esta, se vuelven indispensables en contextos de tránsito y establecimiento migratorio.

c) Asimismo, se ha identificado que la subjetividad y la intersubjetividad, es un elemento de estudio compartido por ambas disciplinas, aunque sostenidas desde teorías distintas. Se entiende desde la sociología al impacto de la sociedad y la cultura en los funcionamientos, pero sobre todo en las conductas de los sujetos. Desde el ámbito psicoanalítico, los procesos internos resultan 
de un intercambio entre dinámicas mentales que no sólo se nutren de sensaciones o reacciones producidas por un aparato mental en aislamiento, sino construido a partir de la interacción con un medio y con otros sujetos. El rescate de lo visible a través de la conducta, pero comprensible a través de la exploración psicoanalítica, vuelve a la complementariedad una propuesta de expansión en el conocimiento del fenómeno migratorio. Desde la sociología, el rescate del fenómeno migratorio como compartido desde una realidad social, pero con fuertes componentes subjetivos que le determinan. Se identifica una suerte de pase de estafeta, donde la perspectiva de la sociología entregue insumos sociológicos que, de manera profunda y desde su complejidad, sean analizados desde una perspectiva psicoanalítica. Con esto se rescata a la subjetividad como una realidad relevante y que dirige a los sujetos. Entendiendo que, aunque un sujeto muestre una conducta manifiesta, los motivos latentes pueden estar lejos de ser congruentes con la acción.

d) Durante la exploración sobre los fundamentos epistemológicos, tanto en los estudios de migración como en los psicoanalíticos, se identificó un intercambio entre lo considerado como explicación y comprensión, es decir, una aproximación a los fenómenos humanos desde fundamentos que privilegian tanto el conocimiento adquirido empíricamente como a través de los significados y la empatía con los sujetos de estudio (comprendiéndoles como personas a partir de la familiarización con el contexto de sus significados y entendiéndoles como sujetos que interpretan la realidad que viven). Tanto los fundadores de los estudios sociológicos, como los psicoanalíticos, no se establecieron totalmente en los postulados de una tradición exclusiva, sino que más bien, se ajustaron a los hechos del fenómeno, reflejándose un intercambio de variables tanto cuantitativas como cualitativas, aunque destacándose una, según fuera el caso. Se considera que esto obedece a la actual categorización de estudios mixtos en investigación, vía a través de la cual, la complementación de ambas disciplinas en los estudios de migración se vuelve viable.

e) Por otro lado, se identificó que en los estudios psicoanalíticos se ha venido problematizando la necesidad de integrar metodologías que nutran a partir de estudios empíricos. Por lo tanto, las exploraciones empíricas de los estudios de migración pueden encontrar en esta problematización, una posibilidad de complementación con los estudios psicoanalíticos en apertura y reconociendo la valía de dichos hallazgos.

f) Se considera relevante puntualizar que, congruente con su epistemología, los estudios psicoanalíticos pueden partir de elementos empíricos o conductuales en los sujetos, sin embargo, este no es su fin. Su escucha y análisis encuentra solo un primer paso en esa realidad para, a través de un encuadre específico, entender planos que no resultan viables de percepción a través de la mera observación. La escucha más allá de lo literal o manifiesto y dirigida hacia lo latente, la comprensión, la deducción y la interpretación, se vuelve un ejerció viable de integración con los estudios de migración que pretendan comprender aspectos inherentes y ocultos en la acción de migrar, buscando un conocimiento sobre mecanismos inconscientes que van más allá de la empírea o la objetividad.

g) Por lo tanto, la propuesta recae en una metodología complementaria que integre elementos socioculturales en la exploración, pero que, a su vez, entienda al 
fenómeno migratorio como un evento social que no solo tiene repercusiones en el momento actual del sujeto migrante que se adapta a una sociedad (entendiéndolo en el tercer momento del proceso o postmigración), sino que, además, tiene importantes antecedentes mentales que pueden brindar una importante orientación y comprensión del fenómeno.

Finalmente, se proponen algunos elementos específicos a manera de sugerencia para tomar en cuenta al momento de realizar la complementación entre ambas disciplinas:

1. Evitar, en el plano de la investigación, la clásica concepción de roles clínicos a partir de la sanidad-enfermedad que parte de los estudios clínico-médico, en los que se busca una cura. Se propone más bien, un conocimiento que lleve a la claridad y comprensión de los motivos que llevan al encuentro del investigador y sujeto de estudio, esto en el sentido planteado por Devereux (1967). Esto significa que el investigador se cuestione sobre el por qué investiga tal o cual fenómeno y por qué elige ciertos sujetos de estudio, pero esto, más allá de su planteamiento del problema en términos académicos, es decir, asumiéndose como un sujeto con motivaciones y dinámicas mentales como cualquier persona; motivaciones que le pueden llevar a intelectualizar problemas o fenómenos asociados a su propia personalidad.

2. Definir de manera clara el desarrollo teórico en psicoanálisis en que se sostiene la investigación complementaria por realizarse, asumiendo que, dentro de los mismos estudios psicoanalíticos, existen diferentes desarrollos teóricos que no siempre son coincidentes, por ejemplo, el freudiano, el kleiniano, el lacaniano, entre otros. Esto vendría a exigir conocimientos fundamentados en teoría y técnica psicoanalítica y no un conocimiento ligero sobre la teoría freudiana o psicoanalítica de manera general. Lo anterior permitirá definir los elementos específicos y fundamentales del funcionamiento psíquico que se persiguen en la investigación, así como su viabilidad.

3. Someter como variable de análisis la convocatoria o acercamiento de los sujetos de estudio a la investigación misma, considerando que existen motivos y figuras inconscientes que motivan las conductas de los sujetos, inclusive cuando participan en una investigación. Esto llevaría a comprender los motivos de la participación y si existe una vinculación mental en el apoyo con el estudio.

4. Relacionado con el punto anterior, se vuelve una posibilidad el análisis de la variable de gratificación que puede obtener el sujeto que colabora en la investigación como elemento operante. Es conveniente reflexionar sobre la ganancia o experiencia cuando aportan y comparten su historia, sus crisis y su funcionamiento mental. Momento en que, a través de una investigación, exponen experiencias traumáticas o dolorosas, frente a un estudio social que únicamente se plantea el buscar información. Esto llevaría a analizar, tanto la influencia de la presencia o ausencia de una gratificación (económica, de atención, etc.) y su impacto en las narrativas de los sujetos, así como la manera en que esta dinámica influye en la percepción que tienen sobre el investigador y quien promueve el estudio. 


\section{Reflexiones finales}

La integración de dinámicas subjetivas en los estudios de migración con base sociológica se considera relevante y necesaria, así como también, la humanización de los sujetos en las investigaciones y el rescate de la comprensión a un nivel empático. En términos epistemológicos y en el plano de la investigación, resulta sumamente relevante el tener definidos los tipos de conocimientos y datos que se indaguen, por lo que la claridad en cuanto a los fines explicativos y/o comprensivos que se busquen en los estudios definirá la ruta a seguir. Sin embargo, tanto en sociología como en psicoanálisis, tal diferenciación dicotómica no ha sido radical, ya que ambas disciplinas les han integrado en sus conocimientos.

En sociología, destacan los fundamentos de Emilio Durkheim y Max Weber, quienes, a pesar de sostenerse desde el actualmente denominado paradigma cuantitativo y cualitativo respectivamente, no descartaron elementos de naturaleza contraria en sus investigaciones, concluyendo que la complejidad de los fenómenos sociales exige ambos tipos de datos y exploraciones. Situación similar ha venido atravesando el psicoanálisis respecto a la consolidación de su objeto de estudio, en una ruta que va desde lo cuantitativo hasta la integración mayoritaria de elementos cualitativos. Por lo tanto, se deduce que ninguno de estos dos paradigmas le contiene completamente, pues en la teoría destacan ambos.

De esta manera, la vinculación entre los estudios de migración con base sociológica y los estudios psicoanalíticos, se vuelve viable cuando se considera que ambas disciplinas buscan tanto elementos conductuales y patrones identificables en los sujetos, como elementos subjetivos viables de interpretación y con significaciones particulares. Además, vale destacarse que es posible alcanzar una mayor comprensión sobre un funcionamiento mental cuando se asume el impacto de las condiciones socioculturales en la construcción de la mente. Por otro lado, la primacía de la escucha y la búsqueda de la humanización de las personas que participan en las investigaciones, son elementos coincidentes en ambas disciplinas, pudiéndose integrar a su vez, la figura del investigador en la dinámica del proceso frente a otro sujeto en el escenario de la investigación, lo cual es un elemento de larga tradición en los estudios psicoanalíticos.

Finalmente, se presentó una propuesta de aproximación metodológica en la complementariedad, donde se destacaron elementos aplicables a los estudios de migración. Estos fueron la integración de elementos socioculturales y psicológicos en la exploración, así como el evitar una categorización clínico-médica e integración de los motivos que llevan al encuentro entre investigador y sujeto de estudio. También se destacó la determinación de la teoría que guiará el estudio, aun cuando por un lado se parta del psicoanálisis, entendiendo que existen diferentes desarrollos teóricos internos en psicoanálisis que han sido parte de sus fundamentos teóricos.

Esto llevaría a evitar planteamientos superficiales que se basan en lecturas teóricas ligeras o que parten exclusivamente de la experiencia en la práctica. Esta definición de enfoque planteada, llevaría a definir el desarrollo teórico por utilizarse, revisando su coincidencia con los fines buscados en la investigación con personas migrantes. Por otro lado, el analizar las variables de convocatoria y los motivos que empujan a un sujeto para participar en un estudio, así como del investigador como sujeto con motivación propia para el mismo, se consideran elementos importantes de consideración. Finalmente, 
el reparo en la variable de gratificación, es decir, reflexionar sobre la ganancia que tienen los sujetos de estudio cuando aportan y comparten su funcionamiento mental, exponiendo experiencias traumáticas o dolorosas, frente a un estudio que busca la información y no su cura, se considera viable de análisis.

La propuesta aquí presentada puede resultar como punto de partida para revisiones más profundas respecto a la complementariedad de ambas disciplinas, así como de referencia para estudios de migración que puedan ahondar en la dinámica subjetiva de los sujetos con base en la teoría psicoanalítica.

\section{Referencias}

Achotegui, J. (2004). Emigrar en situación extrema. El síndrome del inmigrante con estrés crónico y múltiple (síndrome de Ulises). Revista Norte de Salud Mental, $v(21), 39-52$.

Alvarado, R. (2008). Salud mental en inmigrantes. Revista Chilena de Salud Pública, 12(1), 37-41.

Althusser, L. (1965). Freud y Lacan. España: Editorial Anagrama.

Andolfi, M. (2009). La psiocoterapia como viaje transcultural. Revista Psicoperspectivas, $\operatorname{VIII}(1), 6-44$.

Arfuch, L. (2005). Identidades, sujetos y subjetividades. Buenos Aires: Prometeo.

Ariza, M. y Velasco, L. (2015). Métodos cualitativos y su aplicación empírica. México: Instituto de investigaciones sociales, UnAm; El Colegio de la Frontera Norte.

Bleichmar, N. M. y Leiberman, C. (1989). El psicoanálisis después de Freud. México: Editorial Paidós Mexicana.

Bojorquez, I., Aguilera, R. M., Ramírez, J., Cerecero, D. y Mejía, S. (2015). Common mental disorders at the time of deportation: A survey at the Mexico-United States border. Journal of Immigration and Minority Health, 17, 1732-1738. doi: http://10.1007/s10903-014-0083

Bhugra, D. (2004). Migration and mental health. Acta Psychiatrica Scandinavica, 109(4), 243-258.

Bustamante, J. (1997). Cruzar la línea. México: Fondo de Cultura Económica.

Caruso, I. (1979). Aspectos sociales del psicoanálisis. México: Premia Editora.

Creswell, J. W. (2009). Research Design Qualitative, Quantitative, and Mixed Methods Approaches. Estados Unidos: Thousand Oaks, CA Sage Publications.

Criado, M. (1999). La línea quebrada. Historias de vida de migrantes (Tesis doctoral). Universidad Complutense de Madrid, España.

Devereux, G. (1967). De la ansiedad al método en las ciencias del comportamiento. México: Siglo XXI.

Durkheim, E. (1895). Las reglas del método sociológico. México: Fondo de Cultura Económica.

Durkheim, E. (1897). El suicidio. España: Editorial Losada.

Falicov, C. (2001). Migración: Pérdida ambigua y rituales. Perspectivas Sistémicas, (69). Recuperado de http://www.redsistemica.com.ar/migracion2.htm 
Ferrant, C. (2018). Abdelmalek Sayad, sociólogo de la migración Argelia-Francia. Revista Levadura. Recuperado de http://revistalevadura.mx/2018/09/20/abdelmalek-sayad-sociologo-la-migracion-argelia-francia/

Freud, S. (1910). Las perspectivas futuras de la terapia psicoanalítica. Argentina: Editorial Amorrortu.

Germani, G. (1969). La sociología de la modernización. Buenos Aires: Paidós.

Grinberg, L. y Grinberg, R. (1982). Psicoanálisis de la migración y el exilio. España: Paidós.

Heimann, P. (1950). Acerca de la contratransferencia. Recuperado de https://www.jaimeyasky.cl/jaimeyasky/Escritos___Writings_files/Paula\%20Heimann $\% 20$ $\% 281950 \% 29 \% 20$ Acerca\%20de\%20la\%20Contratransferencia.pdf

Herrera, A. (2013). Epistemología del psicoanálisis. Estados Unidos: Palibrio LLC.

Hwang W. C., Myers, H. F., Abe-Kim, J. y Ting, J. Y. (2007). A conceptual paradigm for understanding culture's impact on mental health: the cultural influences on mental health (сімн) model. Clinical Psychology Review, 28(2), 211-27.

Ingleby, D. (2008). New perspectives on migration, ethnicity and schizophrenia. Willy Brandt Series of Working Papers in International Migration and Ethnic Relations 4/07, IMER, MIM, Malmö University, Suecia.

Kachele, H. y Thoma, H. (2003). Teoría y práctica del psicoanálisis. Tomo 3: Investigación. Recuperado de http://www.apdeba.org/wp-content/uploads/tomo_3_completo.pdf

Lee, E. (1966). A theory of migration. Demography, 3(1), 47-57.

Moro, M. R. (2004). ¿Por qué crear dispositivos específicos para los inmigrantes y sus niños? La experiencia francesa. Psicopatología y Salud Mental, 4, 69-80.

Perrés, J. (1988). El nacimiento del psicoanálisis. Apuntes críticos para una delimitación epistemológica. México: Editorial Plaza y Valdés.

Piastro, J. (2015). Los procesos subjetivos de la migración en la obra literaria de Tahar Ben Jelloun. Migraciones Internacionales, 8(1), 221-241.

Racker, H. (1986). Estudios sobre técnica psicoanalítica. Argentina: Editorial Paidós Argentina.

Ricoeur, P. (1969). El conflicto de las interpretaciones. México: Fondo de Cultura Económica.

Sarzuri-Lima, M. (2013). La construcción del "sueño español”. Revista Integra Educativa, $v(1), 127-140$.

Shedler, J. (2010). The efficacy of psychodynamic psychoterapy. American Psychologycal, 65(2), 98-109.

Tarrés, M. (Coord.). (2013). Observar, escuchar y comprender, sobre la tradición cualitativa en la investigación social. México: El Colegio de México.

Temores-Alcantara, G. Infante, C., Caballero, M., Flores-Palacios, F. y Santillanes-Allende, N. (2015). Salud mental de migrantes centroamericanos indocumentados en tránsito por la frontera sur de México. Revista Salud Pública de México, 57(3), 227-233.

Valles, M. (2009). Metodología biográfica y experiencia migratoria: actualidad del enfoque de los testimonios anónimos y de autor en el legado de Juan F. Marsal. Papers: Revista de Sociología, 91, 103-125.

Venturini, A. (2006). La emergencia del sujeto en la migración. Aesthethika. Revista Internacional de Estudio e Investigación Interdisciplinaria sobre Subjetividad, Política y Arte, 2(2), 62-68. 
Vilar, E. y Eibenschutz, C. (2007). Migración y salud mental: un problema emergente de salud pública. Revista Gerencia y Políticas de Salud, 6(13), 11-32.

Weber, M. (1922). Economía y sociedad. México: Fondo de Cultura Económica.

Weber, M. (1958). Ensayos sobre metodología sociológica. Argentina: Editorial Amorrortu.

Ángel Luna

Mexicano. Doctor en Estudios de Migración por el Colegio de la Frontera Norte. Es maestro en psicoterapia psicoanalítica por la Universidad Intercontinental y licenciado en psicología por la Universidad Autónoma de Baja California. Líneas de investigación: complementación de los estudios psicoanalíticos con los estudios de migración desde una perspectiva sociológica, así como el estudio de la salud mental en el plano migratorio.

Silvía Mejia-Arango

Colombiana. Doctora en Psicología por la Universidad Nacional Autónoma de México. Profesora investigadora de El Colegio de la Frontera Norte. Líneas de investigación: salud mental de los adultos mayores de origen mexicano que viven en México y en los Estados Unidos, flujos migratorios y salud mental en la frontera norte, y población deportada en Tijuana, México. Publicación reciente: Mejia-Arango, S., Saenz, R. y Singelmann, J. (2018). Cognitive decline among the elderly: A comparative analysis of Mexicans in Mexico and the United States. Estados Unidos: Springer Press. 